\title{
Perception of Autism Spectrum Disorder Children by Envisaging Emotions from the Facial Images
}

\author{
T. Lakshmi Praveena, N.V. Muthu Lakshmi
}

\begin{abstract}
Image processing is a rapidly growing technology and is one among the thrust areas of research in Medical Fields, various Engineering disciplines, life Sciences and Scientific applications. Many technical applications have already adopted image processing and it plays a key role in predicting unknown or hidden facts easily and efficiently. Facial image processing is an innovative application of image processing and is being widely used in many applications successfully. Some of the applications are used for person identification, identifying authorized persons, identifying criminals and so on. As we all know that person's emotion shows personality \& behavior, moods where he or she expresses feelings by emotions maximum on face only. Facial expression can also be used in various fields like emotion recognition, market analysis, prediction neurological disorder percentage, psychological problems and so on. So, it has become an emerging research area to study. Neurological disorder is a more complicated disease because it affects both physical body and mental body. In this paper a new methodology is proposed using optimized deep learning methods to predict ASD in children of age 1 to 10 years. Proposed model performance is tested on ASD children and normal children facial image dataset collected from Kaggle datasets and also tested on dataset collected from autism parents' face book group. Convolutional Neural Networks (CNN) is applied on extracted face landmarks using optimization techniques, dropout, batch normalization and parameter updating. Most significant six types of emotions are considered for analysis in predicting ASD children accurately.

Keywords: Autism Spectrum Disorder, Convolutional Neural Networks, Deep Neural Networks, Facial emotion recognition.
\end{abstract}

\section{INTRODUCTION}

Facial image processing is an innovative application of image processing and is being widely used in many applications successfully. Some of the applications are used for person identification, identifying authorized persons, identifying criminals and so on. As we all know that person's emotion shows personality \& behavior, moods where he or she expresses feelings by emotions maximum on face only. Facial expression can also be used in various fields like emotion recognition, market analysis, prediction neurological disorder percentage, psychological problems and so on. So, it has become an emerging research area to study. Neurological disorder is a more complicated disease because it affects both physical body and mental body. Facial emotions help us to recognise the feelings, present status of person and helps us to change our behaviour accordingly.

Revised Manuscript Received on November 25, 2020.

* Correspondence Author

T. Lakshmi Praveena*, Research Scholar, Sri Padmavathi Visvavidyalayam, AP, India, praveenalaxmi1@gmail.com

N. V. Muthu Lakshmi, Assistant Professor, Sri Padmavathi Visvavidyalayam, AP, nvmuthulakshmi@gmail.com

(C) The Authors. Published by Blue Eyes Intelligence Engineering and Sciences Publication (BEIESP). This is an open access article under the CC BY-NC-ND license (http://creativecommons.org/licenses/by-nc-nd/4.0/)
So, the ability of producing facial expression is known as the social communication ability and interaction ability [4].Autism spectrum disorder (ASD) is a neurological disorder problem, and it comes by birth. If it is detected at early stage, better treatment and training can be given by Doctors, Physiatrist, and parents. This paper deals about how to predict ASD kids by analyzing emotions and feelings from facial expression using Emotion Recognition(ER) techniques and also analysis is made.Analysing emotions is accurate with facial expression from normal people with one or few attempts where as it is very difficult in case of Autism Spectrum Disorder (ASD) children because they could not communicate, may not mingle other than parents and also they may be unable to speak properly. To detect ASD children from children's facial expressions by analysing their emotions, the present research is used. As it is well known that emotions may be shown on face either consciously or subconsciously, so emotion recognition may be more accurate in detecting ASD children than observing behavior or from questionaries' or clinical or scanning reports. ASD individual has repetitive behaviors, lack of social communication. In ASD individuals identifying and understanding of facial emotions is difficult and ASD individuals also face difficulty in understanding facial emotions in opposite people who are communicating with them. So, the emerging technologies are simplifying this for ASD individuals [5]. Recent studies say that recognizing facial emotions in ASD individuals is also difficult [6]. Research on face processing of ASD individuals proved that ASD people are less responsive in upper part of the face i.e. they are neutral in eyes region while expressing emotions in face [7]. So the lower part of face is very important in recognizing emotion in ASD people, mouth, chin, jaw and cheeks. Kris Evers et.al [8] perform research on lower part of face for recognizing emotion in ASD people by generating hybrid faces. In this paper ASD prediction model is proposed by analysing deep features of face to recognize the facial emotion and to predict ASD in individuals. Dataset used in this paper is collected from Kaggle. Dataset has total 1857 images of ASD and 1850 images of Non-ASD of 6 different facial emotions like happy, angry, fear, normal, surprise and sad.Convolutional Neural Networks (CNN) is employed over extracted face landmarks using optimization techniques, dropout, batch normalization and parameter updating. Most significant six types of emotions are considered for analysis in predicting ASD children accurately. Remaining paper is organized into 4 sections. Section 2 provides literature survey related to ASD and emotion recognition.

Blue Eyes Intelligence Engineering and Sciences Publication

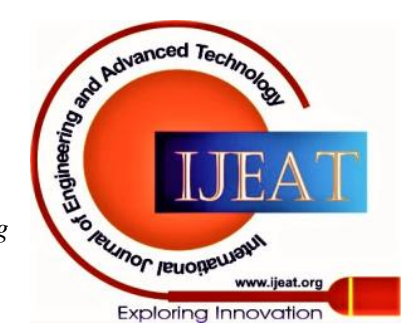




\section{Perception of Autism Spectrum Disorder Children by Envisaging Emotions from the Facial Images}

Section 3 explains about proposed model and architecture of model. Section 4 is the implementation and results of proposed model.

\section{LITERATURE SURVEY}

ASD is a neurodevelopmental disorder which affects behavioral properties of children and social communication features of children [9]. Diagnosing ASD is comparatively difficult than diagnosing other diseases. Because there is no clinical test for ASD. The two factors that affect in diagnosing ASD are 1) The wide range of symptoms and types, 2) the behavioral properties depend on non ASD behavior properties like cognitive and activities [10]. Facial attributes like emotions, arousal and action units also used as biomarkers in predicting ASD [11].

EmotioNet and AffectNet facial emotion image datasets are released in 2017. These large-scale datasets make possible to $\mathrm{CNN}$ to train and predict emotion $[12,13]$. ASD does not has large scale dataset which makes difficulty in applying CNN specially to train with ASD data. The dataset used in present research is received from kaggle.com and dataset author is Gerry [1] who worked for one year to collect 1857 ASD and 1850 TD images from different web resources.

Face Detection is the process of detecting facial regions in face and drawing rectangle for the face region. This process is performed by using haar cascade classifier and viola jones algorithm of face detection [14]. Face Recognition is the process of recognizing the face from a database of face images. Face recognition process uses the landmarks of face using the frontal face predictor algorithm. Dlib library of OpenCV provides 68 landmarks predictor which helps to recognize face, extract features of face and also used in emotion recognition [15,18]. Machine Learning (ML) algorithms provide single label classification models, the deep learning models are efficient to work with multiple label classification.

Recent studies state that facial emotions, facial attributes, and facial features plays an important role in predicting ASD and understanding the behavior of ASD children [4,5]. With advancements in the technology like Artificial Intelligence, machine learning and deep learning improving research on ASD. Egger et.al performed research on head pose and expression analysis to analyze ASD in children [16]. Rudovic et al. studied on facial landmarks and position of body combined with audio and bio-signals to study the state of ASD children and behavior of ASD children [17].

\section{PROPOSED MODEL}

\section{A. Dataset Description}

Dataset used for this research is collected from Kaggle.com datasets. Dataset is uploaded by Gerry piosenka [1] in April 2020. Dataset contains facial images of autistic $(n=1857)$ and non-autistic $(\mathrm{n}=1850)$ children with different expressions. The images are RGB images of dimension $224 \times 224 x 3$. Dataset contains images of age group 1 year to 10 years of both boys and girls. Dataset is divided into training set, test set and validation set with 1666 images for training, 100 images for test and 50 images for validation.

\section{B. Proposed model architecture}

Proposed model has four stages, and the process is given in algorithm as follows:

1. Initial stage:

a. pre-processing stage which accepts images from dataset

b. Resizes images, apply sobel filtering

c. Divide dataset to training and test sets.

2. Face Detection stage:

a. Converts images to gray scale images

b. Detects face region based on haar cascade classifier.

3. Feature Extraction stage:

a. Extracts required features to predict ASD and emotions from images.

4. Deep Neural Network model for predicting ASD/NonASD.Fig 1 shows model summary of proposed deep learning model with 5 convolutional blocks, each with 2 or 3 convolutional 2D layers and a max pooling layer. The fifth block performs average pooling and is connected to dense and dropout layers.

5. First Deep Neural Network model is for predicting ASD/Non-ASD.Fig 1 shows model summary of deep learning model with 5 convolutional blocks, each with 2 or 3 convolutional 2D layers and a max pooling layer. The fifth block performs average pooling and is connected to dense and dropout layers. The output layer has 2 neurons to predict ASD and Non ASD.

6. Second Deep Neural Network model for predicting facial emotion.Fig 2 shows model summary of deep learning model with 5 convolutional blocks, each with 2 or 3 convolutional 2D layers and a max pooling layer. The fifth block performs average pooling and is connected to dense and dropout layers. The output layer has 6 neurons to predict facial emotion from the same images which are used in first DNN model.

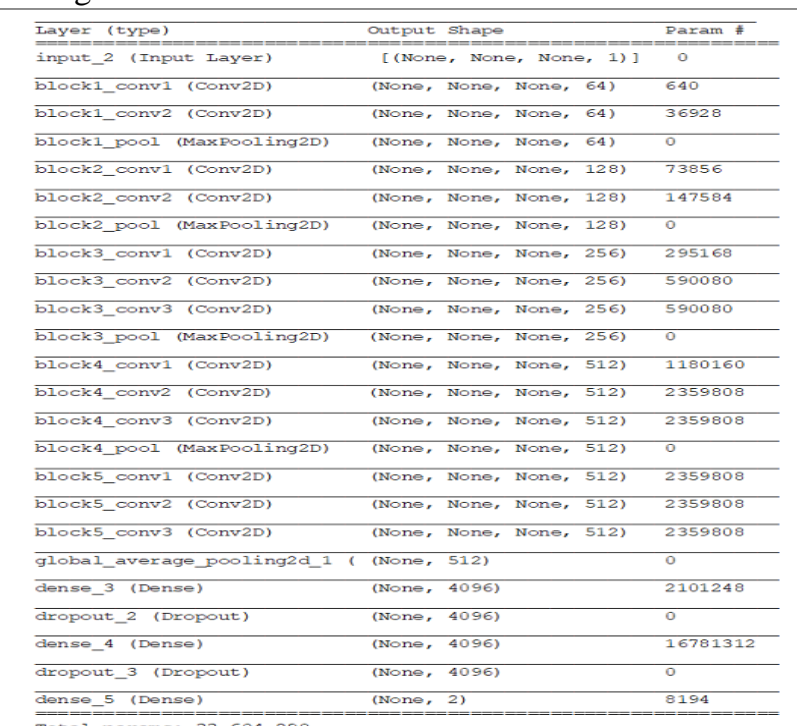

Fig 1.Deep Neural Network model architecture

$$
\text { IV. RESULTS }
$$


The input vectorised data is created with the help of Dlib, OpenCV libraries. The Deep Neural networkmodel is created using Tensor Flow and Keras API in Google Co Lab.

\section{A. Sample result}

Fig 3 gives sample result of emotion prediction model and accuracy of model. The fractional values represent the predicted emotion from images, these values correspond to six emotions used in model. The bold value represents highest percentage of predicted emotion.

Training has completed. Now loading test set to see
how accurate the model is
Model accuracy on Test Set is $40.00 \%$
[5.765913486480713, 0.4000000059604645$]$
Instructions for updating:
Please use Model.predict, which supports generators.
Emotion values
[ $[\mathbf{0 . 6 8 6 7 2 7 7 6} 0.075960810 .063415580 .12819432$
$0.040046770 .00565484]$
$[\mathbf{0 . 7 1 8 7 6 6 5 7} 0.049223540 .004578680 .16637465$
$0.060249560 .00080704]$
$[0.181213720 .01501627 \mathbf{0 . 6 9 3 2 5 7 0 3} 0.08381665$
$0.024327440 .00236885]$
$[\mathbf{0 . 4 0 7 9 0 1 0 5} 0.028827590 .006757930 .33749518$
$0.218145820 .00087247]$
$[\mathbf{0 . 6 6 8 6 3 9 4} 0.192617550 .023656850 .10405558$
$0.007751440 .00327908]]$
ASD status is True

Fig 3. Result of the Emotion prediction model Fig 4 shows comparison of validation and training loss and accuracy.

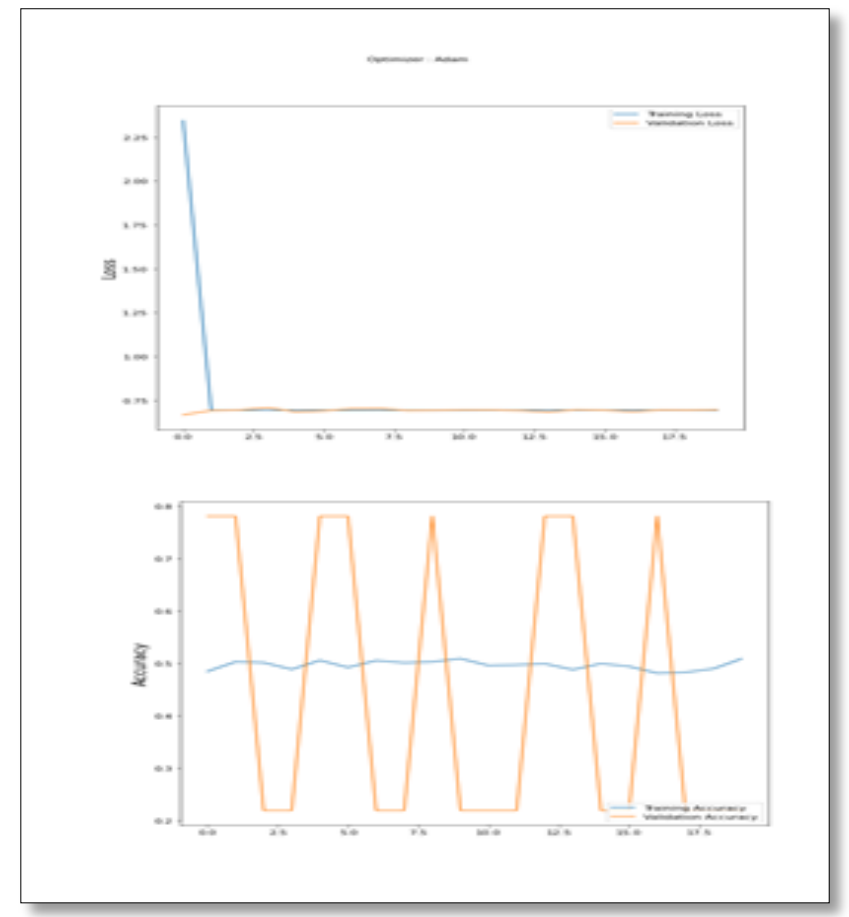

Fig. 4. Accuracy of proposed model validation and training.
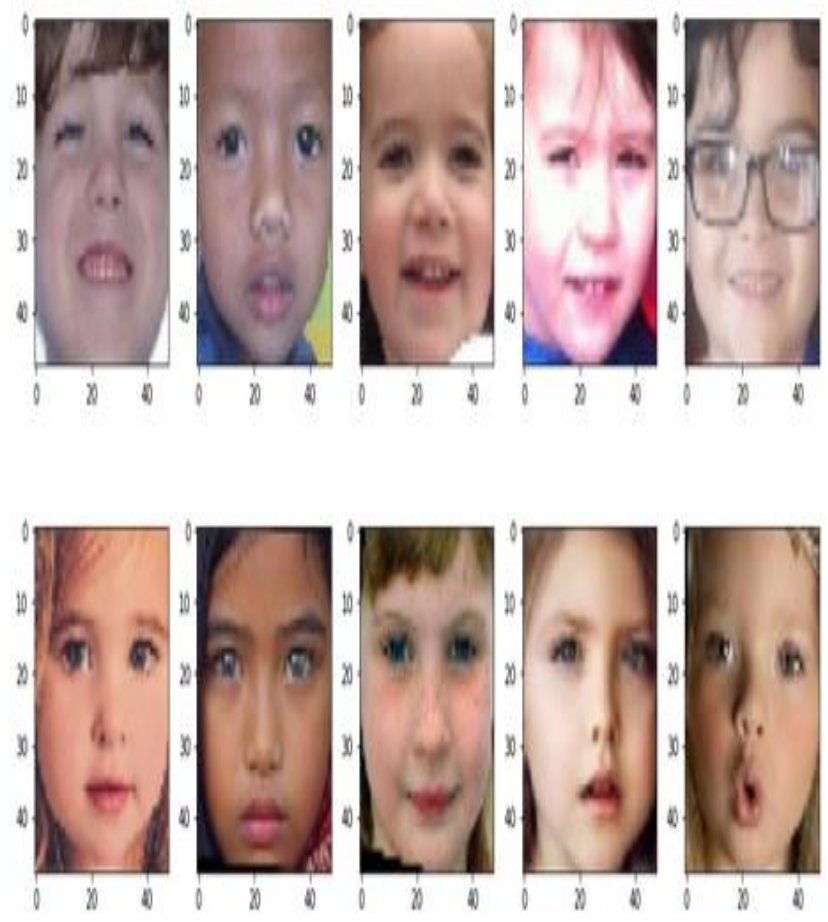

Fig. 5. Autistic and Non Autistic classification based on facial emotions

\section{B. Result of proposed model:}

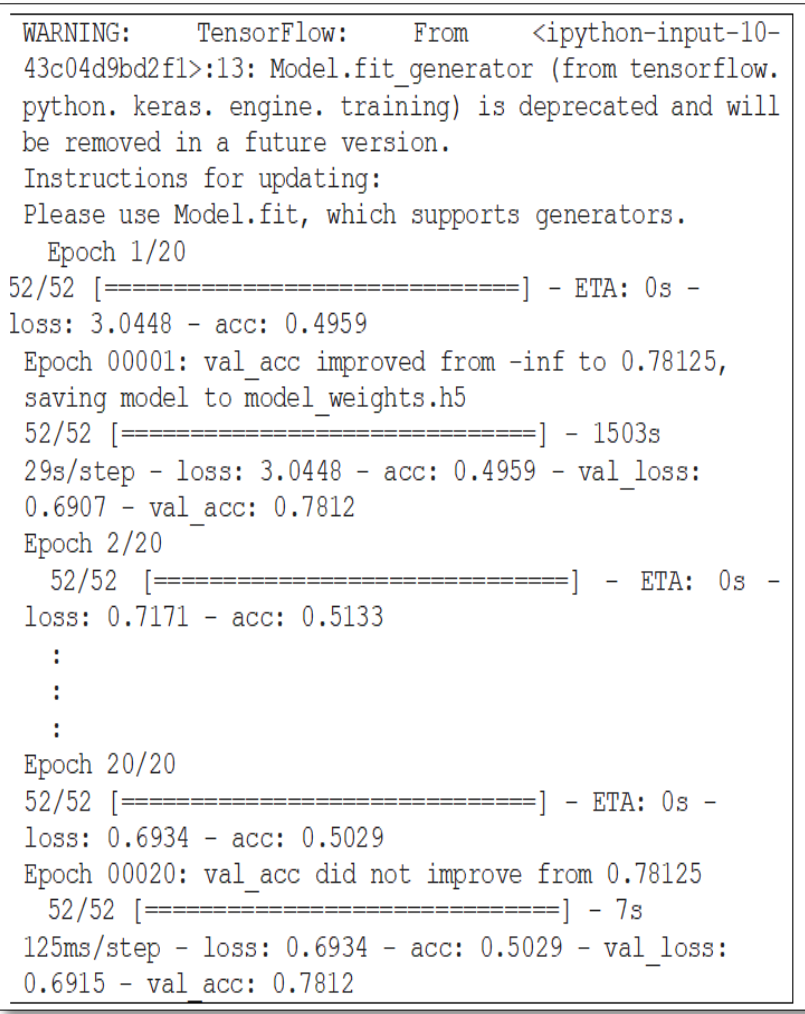

Fig 6. Executed result of proposed model

Table 2 shows sample predicted results of multi label classification for ASD and emotion.

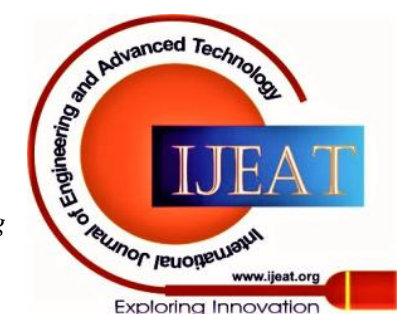


Table 2: Predicted results of multi label classification

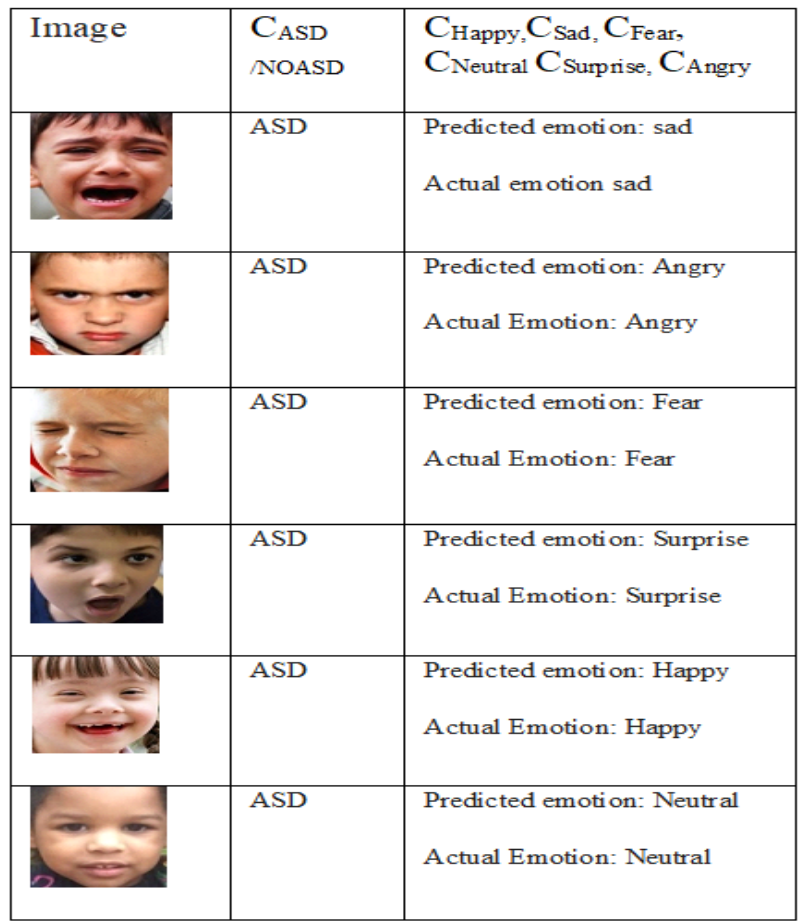

\section{COMPARITIVE RESULTS}

Proposed model is compared with other DNN models with varying of hidden layer blocks. The proposed model is designed with five hidden layer blocks, each block has two 2D convolutional layers and one max pooling layer. Building model with increasing blocks improved accuracy and performance. Model loss reduced comparatively with accuracy. The following table gives the comparative analysis of the model.

Table 3. Comparative analysis of proposed model

\begin{tabular}{|l|l|l|l|}
\hline $\begin{array}{l}\text { Bloc } \\
\text { ks }\end{array}$ & $\begin{array}{l}\text { Number of } \\
\text { neurons }\end{array}$ & Loss & Accuracy \\
\hline 1 & 64 & $\begin{array}{l}0.65508085489 \\
27307\end{array}$ & $\begin{array}{l}0.63999998569 \\
48853\end{array}$ \\
\hline 2 & 64,128 & $\begin{array}{l}0.69319760799 \\
40796\end{array}$ & $\begin{array}{l}0.50499999523 \\
16284\end{array}$ \\
\hline 3 & $64,128,256$ & $\begin{array}{l}0.69315832853 \\
31726\end{array}$ & 0.5 \\
\hline 4 & $64,128,256,51$ & $\begin{array}{l}0.69316124916 \\
07666\end{array}$ & 0.5 \\
\hline 5 & 2 & $\begin{array}{l}0.69316124916 \\
07666\end{array}$ & 0.5 \\
\hline
\end{tabular}

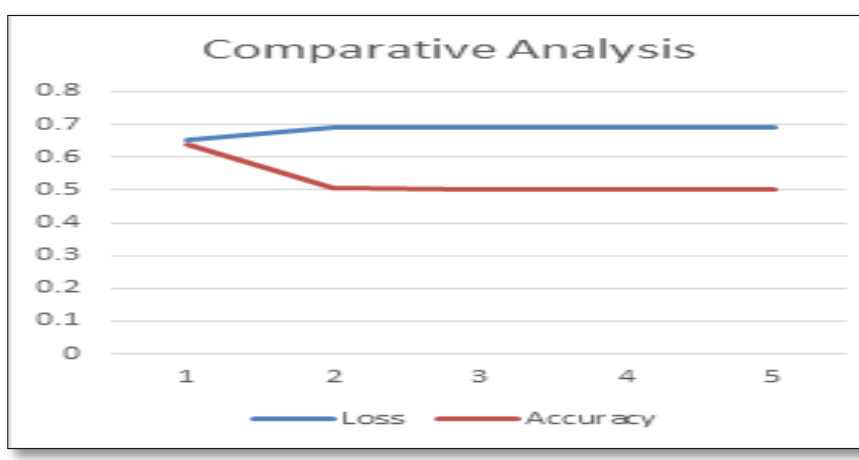

Fig 7 Comparative analysis of model with varying hidden layers
VI.

\section{CONCLUSION AND FUTURE WORK}

Present paper proposed a Deep Neural Network model with multi label classification to predict ASD/NoASD based on facial emotion in ASD and NoASD children. Proposed model is more effective and reliable. The model can also be used to extract different features and attributes in facial images of autism children like action units, arousal, and valence. Facial attributes play an important role in predicting ASD and understand the behaviour of children.

\section{ACKNOWLEDGEMENT}

The dataset used in this paper is collected from web sources [1]. I am thankful to the author of dataset. I prefer this dataset to other autism researchers.

\section{REFERENCES}

1. Gerry (2020, May). Autism Children Data Set, Version 9. Retrieved May 20, 2020 from https://www.kaggle.com/gpiosenka/ autisticchildren-data-set-train test validate.

2. P. N. Bellhumer, J. Hespanha, and D. Kriegman. Eigenfaces vs. fisherfaces: Recognition using class specific linear projection. IEEE Transactions on Pattern Analysis and Machine Intelligence, Special Issue on Face Recognition, 17(7):711--720, 1997

3. LoBue, V. \& Thrasher, C. (2015). The Child Affective Facial Expression (CAFE) Set: Validity and reliability from untrained adults. Frontiers in Emotion Science, 5.

4. I. Nachson, "On the modularity of face recognition: the riddle of domain specificity," Journal of Clinical and Experimental Neuropsychology, vol. 17, no. 2, pp. 256-275, 1995.

5. American Psychiatric Association, Diagnostic and Statistical Manual of Mental Disorders, Washington, DC, USA, 4th edition, 2000.

6. S. Kuusikko, H. Haapsamo, E. Jansson-Verkasalo et al., "Emotion recognition in children and adolescents with autism spectrum disorders," Journal of Autism and Developmental Disorders, vol. 39, no. 6, pp. 938-945, 2009.

7. S. Weigelt, K. Koldewyn, and N. Kanwisher, "Face identity recognition in autism spectrum disorders: a review of behavioural studies," Neuroscience and Biobehavioral Reviews, vol. 36, no. 3, pp. 1060-1084, 2012

8. Kris Evers et.al, "No differences in face emotion recognition strategies in children with ASD: Evidence from hybrid faces", Hindawi publications, Autism research and Treatment , Volume 2014, Article ID 345878, 8 pages, http://dx.doi.org/10.1155/2014/345878.

9. A Ting Wang, Mirella Dapretto, Ahmad R Hariri, Marian Sigman, and Susan Y Bookheimer, "Neural correlates of facial affect processing in children and adolescents with autism spectrum disorder," Journal of the American Academy of Child \& Adolescent Psychiatry, vol. 43, no. 4, pp. 481-490, 2004.

10. Tony Charman, "Variability in neurodevelopmental disorders: evidence from autism spectrum disorders," in Neurodevelopmental Disorders. 2014.

11. E Loth, L Garrido, J Ahmad, E Watson, A Duff, and B Duchaine, "Facial expression recognition as a candidate marker for autism spectrum disorder: how frequent and severe are deficits?" Molecular autism, 2018.

12. A. Mollahosseini, B. Hasani, and M. H. Mahoor, AffectNet: A database for facial expression, valence, and arousal computing in the wild," IEEE Transactions on Affective Computing, 2018.

13. C Fabian Benitez-Quiroz, Ramprakash Srinivasan, and Aleix M Martinez, "EmotioNet: An accurate, real-time algorithm for the automatic annotation of a million facial expressions in the wild," in Proceedings of the IEEE Conference on Computer Vision and Pattern Recognition, 2016, pp. 5562-5570.

14. Zhanpeng Zhang, Ping Luo, Chen Change Loy, and Xiaoou Tang, "Facial landmark detection by deep multi-task learning," in European Conference on Computer Vision. Springer, 2014, pp. 94-108. 
15. Momin H., Tapamo JR. (2011) Automatic Detection of Face and Facial Landmarks for Face Recognition. In: Kim T., Adeli H., Ramos C., Kang BH. (eds) Signal Processing, Image Processing and Pattern Recognition. SIP 2011. Communications in Computer and Information Science, vol 260. Springer, Berlin, Heidelberg.

16. Helen L Egger, Geraldine Dawson, Jordan Hashemi, Kimberly LH Carpenter, Steven Espinosa, Kathleen Campbell, Samuel Brotkin, Jana Schaich-Borg, Qiang Qiu, Mariano Tepper, et al., "Automatic emotion and attention analysis of young children at home: a researchkit autism feasibility study," npj Digital Medicine, vol. 1, no. 1, pp. 20, 2018.

17. Ognjen Rudovic, Jaeryoung Lee, Miles Dai, Bjorn Schuller, and Rosalind Picard, "Personalized machine learning for robot perception of affect and engagement in autism therapy," Science. Doi: 3.10.1126/scirobotics. aao6760., 2018.

18. de Carvalho A.C.P.L.F., Freitas A.A. (2009) A Tutorial on Multilabel Classification Techniques. In: Abraham A., Hassanien AE., Snášel V. (eds) Foundations of Computational Intelligence Volume 5. Studies in Computational Intelligence, vol 205. Springer, Berlin, Heidelberg.

19. Maxwell, A., Li, R., Yang, B. et al. Deep learning architectures for multi-label classification of intelligent health risk prediction. BMC Bioinformatics 18, 523 (2017). https://doi.org/10.1186/s12859-0171898-z

\section{AUTHORS PROFILE}

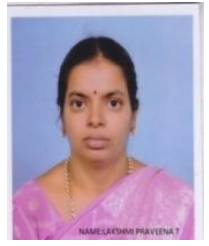

Traveena is a Research scholar at $\mathrm{St}$ Padmavathi Mahila Visvavidyalayam, Tirupati, AP, India. She completed her M. Tech (CSE), B. Tech (CSE) from JNTUK, AP, India. Her research area is data analytics and working on analysis of ASD in children. Other research interests are Data mining. Machine learning and Deep learning.

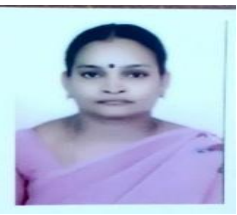

N.V. Muthu Lakshmi is an Assistant Professor at Sri Padmavathi Mahila Visvavidyalayam, Tirupati, AP, India. I have done Ph.D in Sri Padmavati Mahila Visvavidyalayam, Tirupati, INDIA. My Thrust areas are Privacy preserving Data Mining, Association Rule Mining in Centralized and Distributed Environment, Data Analytics, Big data, Cloud Computing, Distributed databases, Machine Learning Algorithms,Network Security 